\title{
Fractal Dimension Calculation of a Manganese-Chromium Bimetallic Nanocomposite Using Image Processing
}

\author{
Amir Lashgari, ${ }^{1}$ Shahriar Ghamami, ${ }^{1}$ Saeedeh Shahbazkhany, \\ Guillermo Salgado-Morán, ${ }^{2}$ and Daniel Glossman-Mitnik ${ }^{3}$ \\ ${ }^{1}$ Department of Chemistry, Faculty of Science, Imam Khomeini International University, Qazvin 34148-96818, Iran \\ ${ }^{2}$ Departamento de Ciencias Químicas, Facultad de Ciencias Exactas, Universidad Andrés Bello, Sede Concepción, \\ 4070000 Concepción, Chile \\ ${ }^{3}$ Laboratorio Virtual NANOCOSMOS, Departamento de Medio Ambiente y Energía, \\ Centro de Investigación en Materiales Avanzados (CIMAV), 31136 Chihuahua, CHIH, Mexico
}

Correspondence should be addressed to Daniel Glossman-Mitnik; daniel.glossman@cimav.edu.mx

Received 11 February 2015; Revised 9 April 2015; Accepted 16 April 2015

Academic Editor: Ungyu Paik

Copyright (C) 2015 Amir Lashgari et al. This is an open access article distributed under the Creative Commons Attribution License, which permits unrestricted use, distribution, and reproduction in any medium, provided the original work is properly cited.

Bimetallic materials, which have the ability to convert heat change into mechanical movement, normally consist of two bonded strips of dissimilar metals that expand at different rates. We describe how we made a manganese-chromium (Mn-Cr) bimetallic nanocomposite using the centrifuge method and a low-to-high approach. We conducted scanning electron microscope (SEM) imaging, energy-dispersive X-ray spectroscopy (EDX) analysis, and X-ray diffraction spectra of the nanocomposite to prove its identity. We examined how centrifuge speed, process time, and the use of an "intruder agent" affected the properties of the material. The fractal dimension is a significant factor that can be used to approximate the surface roughness, the texture segmentation, and an image of the studied compounds. We calculated the technique of fractal dimensions using image-processing values on a computer and histogram plot with the SEM image of the Mn-Cr bimetallic nanocomposite using MATLAB software. We applied the Statistical Package for the Social Sciences software for statistics data extracted from the SEM image of the nanocomposite and obtained the following results: mean $=1.778$, median $=1.770, \max =1.98, \min =1.60$, skewness $=0.177$, range $=0.38$, and harmonic mean $=1.771$ for fractal dimension of the SEM image.

\section{Introduction}

Metal matrix composites (MMCs) are an important engineering material due to their various applications. Lightweight MMCs, due to their significant hardness and strength, have found wide applications in the aerospace and automotive industries. However, these applications have remained limited due to the lack of stretch of the composites. Composite conversion nanocomposites increase the yield strength and remove these limitations $[1,2]$. MMCs have steadily become more favored in recent years for their high strength and stress resistance properties [3-11].

Chromium ( $\mathrm{Cr}$ ) and chromium compounds are widely used in many industrial procedures such as textile dyeing, printing inks, electroplating, leather tanning, chromium plating, and pigments as serious manufacturing resources
$[12,13]$. Manganese $(\mathrm{Mn})$ is a mineral created in large amounts in both plant and animal tissues. Manganese lowers the temperature at which austenite converts into ferrite, therefore avoiding cementite precipitation at ferrite grain boundaries, and purifies the resulting pearlitic structures. Alloying elements, such as manganese, also enable solutionhardening of ferrite, but this effect is limited compared with that of carbon, nitrogen, phosphorus, and even silicon [14,15].

Image processing of microstructures involves several preparation steps, division, and postprocessing that finally lead to the extraction of quantitative data. Recently, computer-based image-processing methods have advanced quickly, making it possible to quantitatively describe complex colors, shapes, texture properties, and sizes. Imageprocessing methods are regularly used in tandem with mechanical and instrumental devices to change human 
manipulative work in the show of an assumed process $[16,17]$.

The term "fractal dimension" has been used to describe several aspects of particle morphology. The fractal dimension of an individual particle, which can be a main particle or a composite particle such as a floc or aggregate, denotes the indiscretion and self-similarity of the particle. The fractal dimension parameter is determined by computing the length of the predictable perimeter by taking steps of a known length along the border and including the number of ladders. The border length increases with decreasing step size because a smaller step size allows for the presence of more detail in the particle outline [18-20]. The word "fractal" is derived from the Latin "fractious" meaning fragmented; a fractal is defined as a mathematical object with a fractional (noninteger) dimension. It seems that wherever there is structural disorder and chaos, fractal geometry can be an efficient way of describing and analyzing it.

Fractal dimension is a very good mathematical method for the study of unequal geometric substances. Fractal dimension can reflect characteristics appearing in nature. The application of fractal system allows us to explain numerous states of fragmenting and branching occurring in ecological, biological, and other systems [21-24]. The output is a binarized image with a one-pixel-wide boundary appropriate to be used for box-counting calculations to approximate the fractal dimension of the boundary. We developed a MATLAB (MathWorks, Natick, Massachusetts, USA) program based on theory to implement the box-counting technique for fractal dimension calculations.

Several specific theoretical and numerical models have been proposed in order to assess the fractal dimension. Recent studies have examined dimensions, but the majority of them were only one- or two-dimensional. These dimensions are a clear limitation to the study of complex and more general strengthening geometries $[25,26]$.

Manganese and chromium have been used in this nanocomposite; potassium permanganate and potassium dichromate were used as the starting materials. Scanning electron microscope (SEM) images of the nanocomposites may be regular or irregular and their fractal dimension can be calculated. It is interesting to compare the fractal images of the nanocomposites. For example, in this paper, we investigated the size of the nanoparticles and calculated their fractal dimension. We also measured the average, minimum, and maximum of fractal dimensions and image's histogram and normalized image's histogram plot of the SEM image [27-29].

\section{Experimental}

2.1. Materials and Instruments. The starting materials were obtained from Merck (Berlin, Germany) and were used without further purification. The surface morphology of the product was characterized by using a LEO-1430VP scanning electron microscope (Zeiss, Germany) with an accelerating voltage of $15 \mathrm{kV}$. X-ray powder diffraction (XRD) measurements were performed using a Philips X'pert diffractometer (PANalytical, Almelo, Netherlands) with monochromatized
$\mathrm{CuK} \alpha$ radiation. The sizes of the selected samples were estimated using the Scherer method. For identification, the SEM samples were coated with gold.

2.2. Synthesis of Nanocomposite Manganese and Chromium. Potassium permanganate $\left(\mathrm{KMnO}_{4}\right)$ and potassium dichromate $\left(\mathrm{K}_{2} \mathrm{Cr}_{2} \mathrm{O}_{7}\right)$ were used as starting materials to prepare this nanocomposite. Initially, $0.2 \mathrm{~g}$ of potassium permanganate was transferred to a test tube and $0.01 \mathrm{~g}$ of clay was added as an intruder agent. Ethanoic acid, $\mathrm{CH}_{3} \mathrm{COOH}$ $(4 \mathrm{~mL})$, was then added to the mixture and the tube was quickly transferred to a centrifuge and run at $3000 \mathrm{rpm}$ for 45 minutes. The sample was then taken out of the centrifuge and put aside. Then, $0.5 \mathrm{~g}$ of potassium dichromate and $0.01 \mathrm{~g}$ of clay were added to another test tube. $\mathrm{NH}_{3}(5 \mathrm{~mL})$ solution was added and the tube was placed in a centrifuge and run at $3000 \mathrm{rpm}$ for 45 minutes.

2.3. Characterization of the Nanocomposite. The XRD technique was used to determine the composition of the milled powder. The morphology and weight and atomic percentage of the nanocomposite were observed using a SEM image and EDX analysis, respectively. The contents of the first tube were thoroughly stirred and transferred to a second tube. This second tube was returned to the centrifuge and run at $3000 \mathrm{rpm}$ for 45 minutes. The test tube was then removed from the centrifuge and the precipitate was separated out using filter paper. After drying, the precipitate was imaged with SEM instrument, and the atomic percentages were achieved by EDX analysis. So, the XRD spectrum was obtained to show that a manganese-chromium nanocomposite had been produced.

\subsection{Calculation of Fractal Dimension and Image Analysis.} Dimension is one of the most significant ideas in fractal geometry. Now, there are numerous descriptions of this quantity, including packing, dimension, box-counting dimension, Hausdorff dimension, and modified box-counting dimension. Since the mathematic design and approximation of the box-counting dimension are straightforward, this quantity has become one of the most commonly used general dimensions [30, 31]. The calculations and analysis were done by programming with MATLAB and Statistical Package for the Social Sciences (SPSS; IBM, Armonk, New York, USA) software [32]. Moreover, by declaring that the calculation results were comparable, all of the images were processed using a similar crop size and threshold value. Using this method, we calculated the fractals dimension theoretically by applying the following formula:

$$
D=\lim _{r \rightarrow 0} \frac{\log N_{r}}{\log (1 / r)},
$$

where $D$ is the fractal dimension and $N_{r}$ is the number of squares including a part of the considered fractal dimensions.

Considering the resulting numbers, we drew a logarithmic diagram in which the vertical and horizontal axes were $\log (1 / r)$ and $\log N_{r}$, respectively, and the coefficient of 


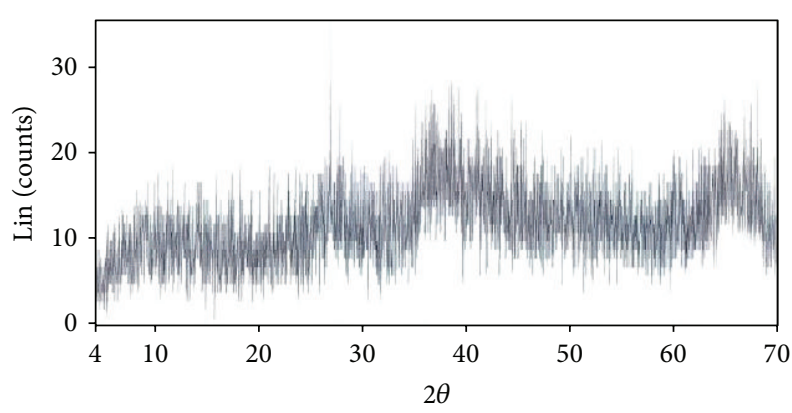

(a)

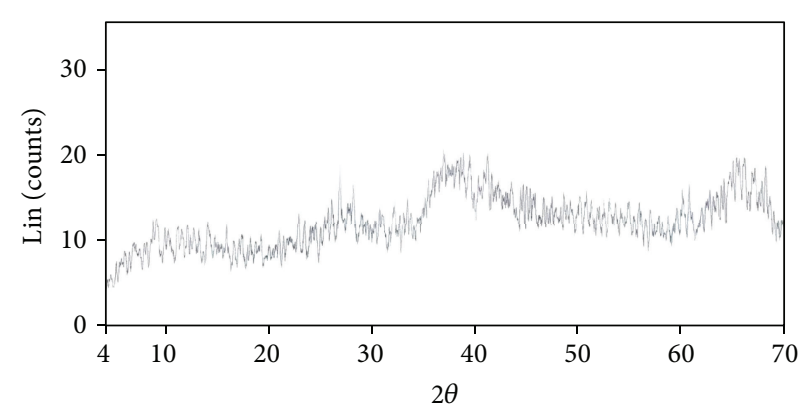

(b)

FIGURE 1: XRD spectrum for the manganese-chromium bimetallic nanocomposite produced via centrifuge: (a) poor crystalline structure (amorphous) and (b) good crystalline structure.

the box size was 1.3. We used a program written in MATLAB to calculate the fractal dimension. There are several methods for calculating the fractal definition including (1) the selfsimilarity definition [33], (2) the mass fractal dimension [34], (3) the spare-sphere fractal dimension [35], (4) the Euclid dimension [36], (5) the grid style [37], (6) the analytical approach for fractal diminishing [38], and (7) the boxcounting fractal dimension [39].

We used the self-similar method for determining the fractal dimension from the SEM image, which employed the box-counting method for the fractal dimension.

For the box-counting fractal dimension we suppose that $F$ is a nonempty and bounded subset of $R^{n}$ and we suppose that $N_{r}(F)$ is the fewest number of collections with a maximum dimension of $(r)$ that can cover $(F)$. The box-counting dimensions under and above $(F)$ : if they are equal we say they are equal to the box-counting dimension or the $F$ box dimension; then we define

$$
\operatorname{dim}_{B} F=\lim _{r \rightarrow 0} \frac{\log N_{r}(F)}{-\log r} .
$$

The circle of cubes in $r$ coordinates from $R^{n}$ implies cubes in the following form:

$$
\begin{aligned}
& \text { If }\left[m_{1} r,\left(m_{1}+1\right) r\right] \times \cdots \times\left[m_{n} r,\left(m_{n}+1\right) r\right], \\
& \text { then } m_{n}, \ldots, m_{1} \text { are integers. } \\
& \text { Finally, } N_{r} \sqrt{n}(F) \leq N_{r}^{\prime}(F) \text {. } \\
& \text { If } r \sqrt{n}<1 \text {, } \\
& \text { then } \log N_{r} \sqrt{n}(F) / \log (r \sqrt{n}) \leq \log N_{r}^{\prime}(F) /(-\log \sqrt{n}- \\
& \log r) \text {. } \\
& \text { If we } \operatorname{limit} \operatorname{dim}_{B} F=\lim _{r \rightarrow 0}\left(\log N_{r}^{\prime}(F) /-\log r\right) \text {, } \\
& \text { then } \overline{\operatorname{dim}}_{B} F \leq \varlimsup_{\lim _{r \rightarrow 0}}\left(\log N_{r}^{\prime}(F) /-\log r\right) \text {. } \\
& \text { Finally, } N_{r}^{\prime}(F) \leq 3^{n} N_{r}(F) \text {. }
\end{aligned}
$$

\section{Results and Discussion}

3.1. Analysis of the Effective Factors for Creating the Nanomaterial. It is important to note that we used centrifuges to make the nanomaterials with the low-to-high method.
After we mixed the solutions they were immediately put into the centrifuge, and an intruder agent was used to ensure that the particles were not connected. This intruder agent must be inert and must not participate in the main reaction. Centrifugal force ensures that the particles produced are not connected and remain on the nanoscale. The advantages of this method are its low cost, rapidity, and ease of application.

Here, we examine the preparation of a manganesechromium bimetallic nanocomposite. The XRD spectrum and SEM image and EDX analysis prepared for the sample both confirmed the creation of a nanocomposite. In the XRD spectrum (Figure 1), no distinguishing peaks are visible and these data did not identify the substance. This outcome is normal because composites are amorphous and their spectra are wide and uniform, unlike the spectra of crystalline materials that have clear and sharp peaks.

The SEM image (Figure 2) shows that the particles are smaller than $100 \mathrm{~nm}$, which also confirms that this material is a nanomaterial. We were therefore able to confirm that we made a manganese-chromium bimetallic nanocomposite. We then obtained 30 random pictures from the SEM image and calculated their fractal dimensions using MATLAB software [40]. For these 30 fractal dimensions we obtained image's histogram and normalized image's histogram, mean, median, max and min, range, skewness, and harmonic mean using SPSS software [41].

The EDX analysis (IDFix report) for the manganesechromium bimetallic nanocomposite is shown in Table 1 and Figure 3. As shown in the IDFix report, the gold present (11.89\%) was used for SEM imaging and was not part of the nanocomposite. The primary elements of the nanocomposite were manganese $(24.33 \%)$, chromium $(3.84 \%)$, carbon $(6.77 \%)$, nitrogen $(2.61 \%)$, potassium $(8.73 \%)$, and oxygen (36.34\%); other elements (silicon $(2.24 \%)$, sulfur $(0.25 \%)$, copper $(2.07 \%)$, and calcium $(0.92 \%)$ ) were related to the clay intruder compound (Figure 3 ). These results confirmed the correct synthesis of nanocomposite.

3.2. Analysis Using MATLAB and SPSS Data. Thirty pictures were selected at random from the SEM image. We first calculated the fractal dimension for these 30 images using MATLAB software (Table 2). Mean, median, max and min, 

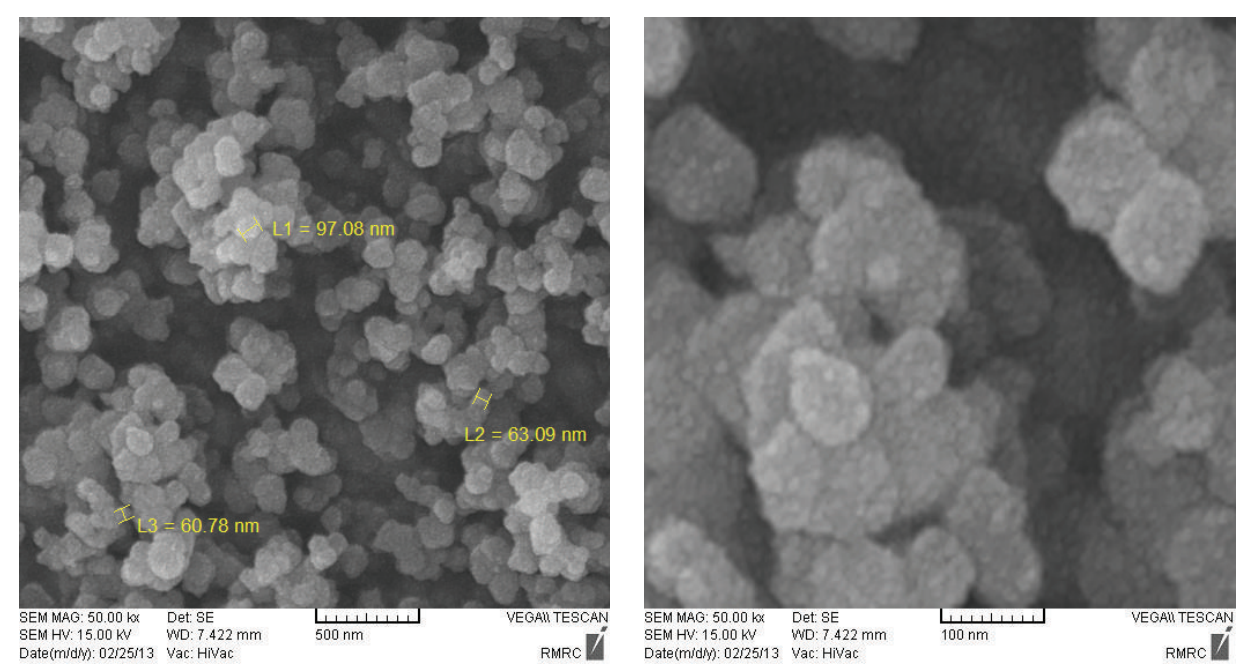

FIGURE 2: SEM images of manganese-chromium bimetallic nanocomposite produced via centrifuge.

TABLE 1: IDFix report for the manganese-chromium bimetallic nanocomposite.

\begin{tabular}{|c|c|c|c|c|c|c|c|}
\hline Elt. $^{a}$ & Int. $^{\mathrm{b}}$ & $W \%^{\mathrm{c}}$ & $A \%^{\mathrm{d}}$ & Elt. $^{a}$ & Int. $^{\mathrm{b}}$ & $W \%^{\mathrm{c}}$ & $A \%^{\mathrm{d}}$ \\
\hline $\mathrm{C}$ & 80.2 & 6.77 & 14.22 & $\mathrm{Ca}$ & 79.8 & 0.92 & 0.58 \\
\hline $\mathrm{N}$ & 17.0 & 2.61 & 4.69 & $\mathrm{Cr}$ & 186.3 & 3.84 & 1.86 \\
\hline $\mathrm{O}$ & 932.4 & 36.34 & 57.29 & $\mathrm{Mn}$ & 973.9 & 24.33 & 11.17 \\
\hline $\mathrm{Si}$ & 312.1 & 2.24 & 2.01 & $\mathrm{Cu}$ & 32.9 & 2.07 & 0.82 \\
\hline$S$ & 30.7 & 0.25 & 0.20 & $\mathrm{Au}$ & 12.1 & 11.89 & 1.52 \\
\hline K & 827.6 & 8.73 & 5.63 & & & & \\
\hline
\end{tabular}

${ }^{\mathrm{a}}$ Element; ${ }^{\mathrm{b}}$ intensity; ${ }^{\mathrm{c}}$ weight percentage; ${ }^{\mathrm{d}}$ atomic percentage.

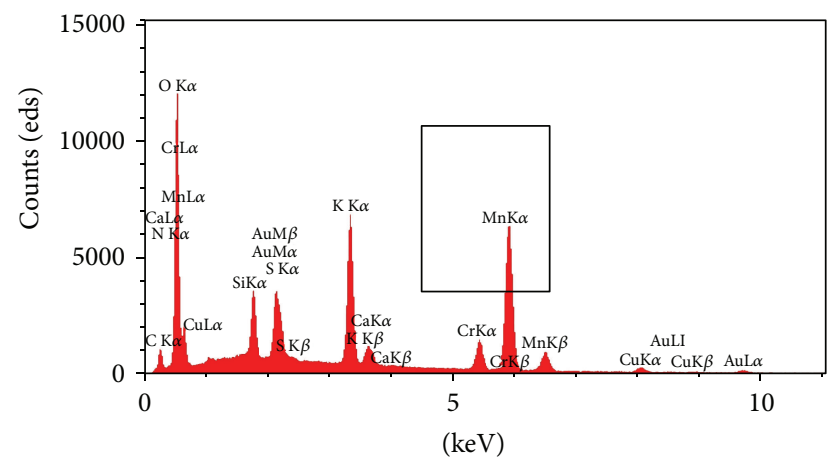

FIGURE 3: IDFix plot for the manganese-chromium bimetallic nanocomposite.

range, skewness, and harmonic mean were also obtained for these data (Table 2) using SPSS software (Table 3). In Table 3, the mean of the fractal dimension is 1.778 , the median is 1.770 , the minimum is 1.60 , the maximum is 1.98 , the range is 0.38 , the skewness is 0.177 , and the harmonic mean is 1.771 .

In statistics and probability theory, the skewness reflects the degree of asymmetry of the probability distribution.
Skewness is a measure of the presence or absence of distribution function symmetry. For a perfectly symmetrical distribution, the skewness is zero; for an asymmetric distribution with stretching toward higher quantities, the skewness is positive. Conversely, for an asymmetric distribution with stretching toward smaller quantities, the skewness is negative. Given that the amount of skewness for particles in this nanocomposite was positive $(+0.177)$, this result reveals an asymmetrical distribution of fractal data with stretching toward higher quantities (Table 3 ). The harmonic mean is a measure of central tendency and is typically calculated when the average of the data is important. The harmonic mean was found to be 1.771 for this nanocomposite (Table 3).

A grayscale digital image consists of separate points of gray tones, or brightness, before incessantly variable tones. A normal image is separated into a number of specific points of brightness, and each of those points is defined via a digital data value. A pixel is the greatest fundamental element of some digital image. Each brightness point is a pixel of the digital image. Essentially, the image histogram shows the distribution of the pixel intensities in the image and is continuously used as a reference. Interactive thresholding can be very effective and afford fast, precise information $[42,43]$. To establish the frequency distribution model for 
TABLE 2: Fractal dimension for 30 random images from the SEM image of the manganese-chromium bimetallic nanocomposite.

\begin{tabular}{|c|c|c|c|c|c|}
\hline Pic. number & Pic. & Fractal dimension & Pic. number & Pic. & Fractal dimension \\
\hline 1 & & 1.84 & 16 & & 1.89 \\
\hline 2 & & 1.73 & 17 & & 1.66 \\
\hline 3 & & 1.69 & 18 & & 1.84 \\
\hline 4 & & 1.75 & 19 & & 1.61 \\
\hline 5 & & 1.6 & 20 & & 1.82 \\
\hline 6 & & 1.76 & 21 & & 1.9 \\
\hline 7 & & 1.84 & 22 & & 1.96 \\
\hline 8 & & 1.77 & 23 & & 1.66 \\
\hline 9 & & 1.82 & 24 & & 1.67 \\
\hline 10 & & 1.94 & 25 & & 1.7 \\
\hline 11 & & 1.86 & 26 & & 1.88 \\
\hline 12 & & 1.69 & 27 & & 1.77 \\
\hline 13 & & 1.95 & 28 & & 1.63 \\
\hline 14 & & 1.65 & 29 & & 1.7 \\
\hline 15 & & 1.78 & 30 & & 1.98 \\
\hline
\end{tabular}

fractal data we plotted image's histogram and normalized image's histogram (Figure 4) by MATLAB software for the fractal data distribution.

The descriptive statistics of the fractal dimension was listed in Table 4. This table shows fractal dimensions of 30 random separated pieces of the SEM image with frequency and valid and cumulative percent of each piece. When dealing with statistical information, we come through numbers. Sometimes, the numbers are repeated. After a value repeated more than once, it is recognized as the frequency of a number. The total frequency of all modules less than the higher class boundary of a quantified class is named the cumulative frequency of that class. Cumulative frequency is found by addition of the frequency of a class interval and the frequencies of the former intervals up to that class interval. This is another way of expressing frequency distribution. Cumulative frequency is used to determine the number of explanations that lie below or above a specific value. The best 
TABLE 3: Analysis of fractal data for the manganese-chromium bimetallic nanocomposite.

\begin{tabular}{lcccccc}
\hline Mean & Median & Minimum & Maximum & Range & Skewness & Harmonic mean \\
\hline 1.7780 & 1.7700 & 1.60 & 1.98 & 0.38 & 0.177 & 1.7713 \\
\hline
\end{tabular}
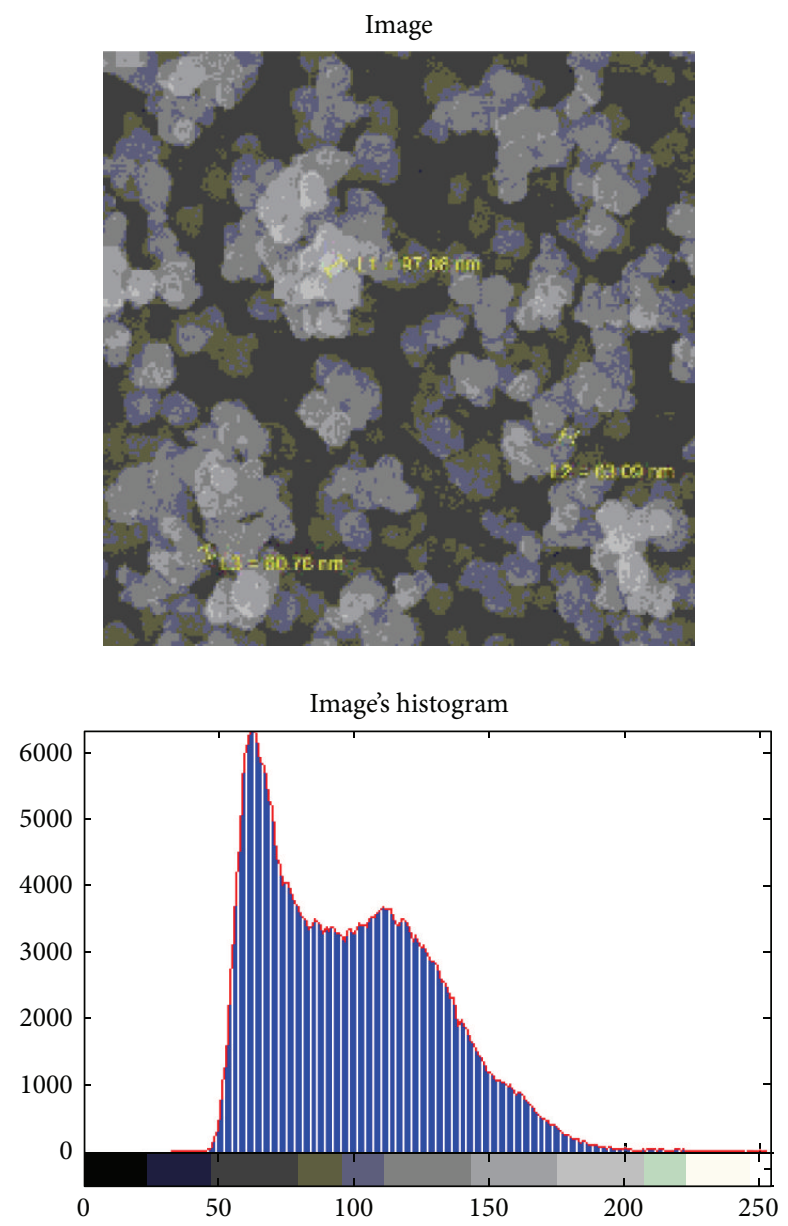
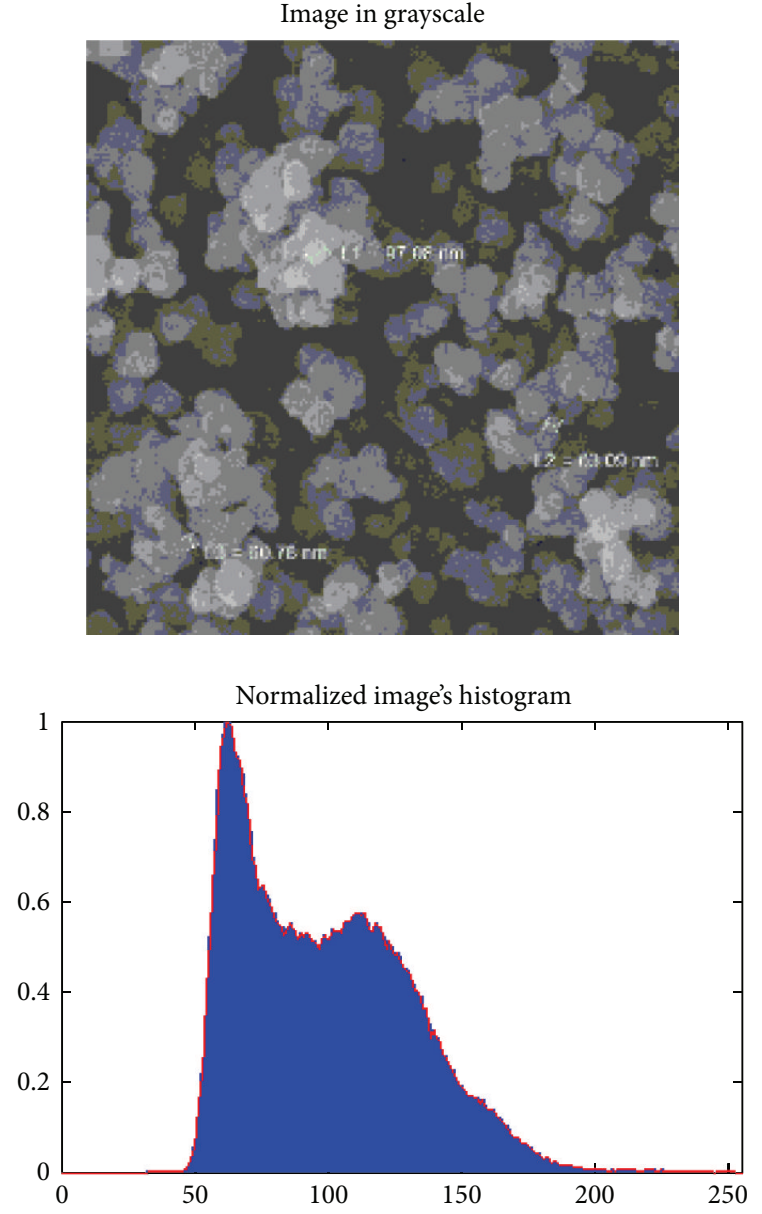

FIGURE 4: Image's histogram and normalized image's histogram of the fractal dimension for the SEM image for the manganese-chromium bimetallic nanocomposite.

advantage of using cumulative percentage is that it offers an easier method to compare different sets of data.

Figure 5 shows a diagram in which there are 30 particles on the horizontal axis and the vertical axis shows the fractal dimension of these particles, which ranges from 1.60 to 1.98. The differences in the fractal dimensions of the particles can be clearly seen, which reveals the nonhomogeneity of the produced particles.

Normal probability plots were generated to check for errors in the normal distribution. If the points of these plots were in a straight line, then normally distributed errors are assumed. According to Figure 6, therefore, which shows a nearly straight line, we conclude that the fractal dimension distribution of the nanocomposite particles is a normally distributed error. Plotting residuals is a useful way of elucidating the goodness of fit of the data. Plotting the residuals versus the corresponding fitted values is a useful model for understanding the common types of disproportion. If

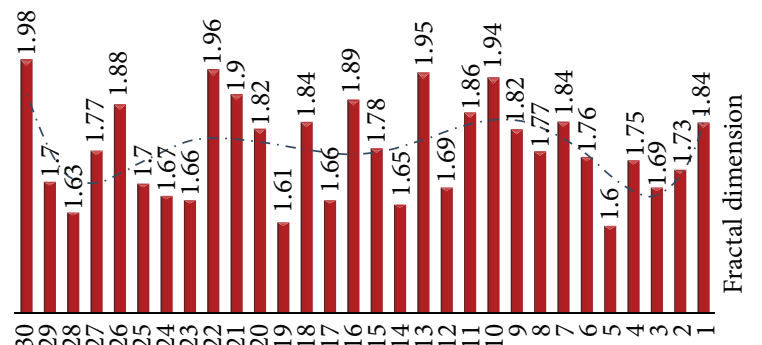

Accidental 30 particles

FIGURE 5: Diagram of 30 particles on the horizontal axis and the amount of fractal dimension on the vertical axis.

the fitted model is appropriate the curve should be symmetrical and the points should be uniformly distributed. Based on Figure 7, one can see that the points are not uniformly 
TABle 4: Descriptive statistics of the fractal dimension of the manganese-chromium bimetallic nanocomposite.

\begin{tabular}{|c|c|c|c|}
\hline \multicolumn{4}{|c|}{ Descriptive statistics } \\
\hline $\begin{array}{l}\text { Fractal } \\
\text { dimension }\end{array}$ & Frequency & Valid percent & $\begin{array}{c}\text { Cumulative } \\
\text { percent }\end{array}$ \\
\hline 1.60 & 1 & 3.3 & 3.3 \\
\hline 1.61 & 1 & 3.3 & 6.7 \\
\hline 1.63 & 1 & 3.3 & 10.0 \\
\hline 1.65 & 1 & 3.3 & 13.3 \\
\hline 1.66 & 2 & 6.7 & 20.0 \\
\hline 1.67 & 1 & 3.3 & 23.3 \\
\hline 1.69 & 2 & 6.7 & 30.0 \\
\hline 1.70 & 2 & 6.7 & 36.7 \\
\hline 1.73 & 1 & 3.3 & 40.0 \\
\hline 1.75 & 1 & 3.3 & 43.3 \\
\hline 1.76 & 1 & 3.3 & 46.7 \\
\hline 1.77 & 2 & 6.7 & 53.3 \\
\hline 1.78 & 1 & 3.3 & 56.7 \\
\hline 1.82 & 2 & 6.7 & 63.3 \\
\hline 1.84 & 3 & 10.0 & 73.3 \\
\hline 1.86 & 1 & 3.3 & 76.7 \\
\hline 1.88 & 1 & 3.3 & 80.0 \\
\hline 1.89 & 1 & 3.3 & 83.3 \\
\hline 1.90 & 1 & 3.3 & 86.7 \\
\hline 1.94 & 1 & 3.3 & 90.0 \\
\hline 1.95 & 1 & 3.3 & 93.3 \\
\hline 1.96 & 1 & 3.3 & 96.7 \\
\hline 1.98 & 1 & 3.3 & 100.0 \\
\hline
\end{tabular}

distributed, revealing an asymmetric curve that conforms to a skewness of 0.177 .

\section{Conclusion}

Our calculations of the fractal dimensions of 30 randomly selected particles from the SEM image of the nanomaterial and the max, min, and range of the data can be used for analyzing the nanoparticles. We considered homogeneity and uniformity of the SEM image by selecting the 30 random particles from it. In fact, the fractal dimension can be obtained from the size and morphology of the particles. If the max and min of the data are close to each other or the range of data is small then we can conclude that the size and dispersion of the particles in the SEM image are similar and that these created nanoparticles show good homogeneity and uniformity. In the SEM image of this nanocomposite the range was large (about 0.38). For this reason, we concluded that the sizes and morphologies of the particles were different. This conclusion was consistent with the SEM image of the nanocomposite. Initially, it seemed that the picture was uniform. However, after doing the calculations it became clear that the produced particles were not homogenous;

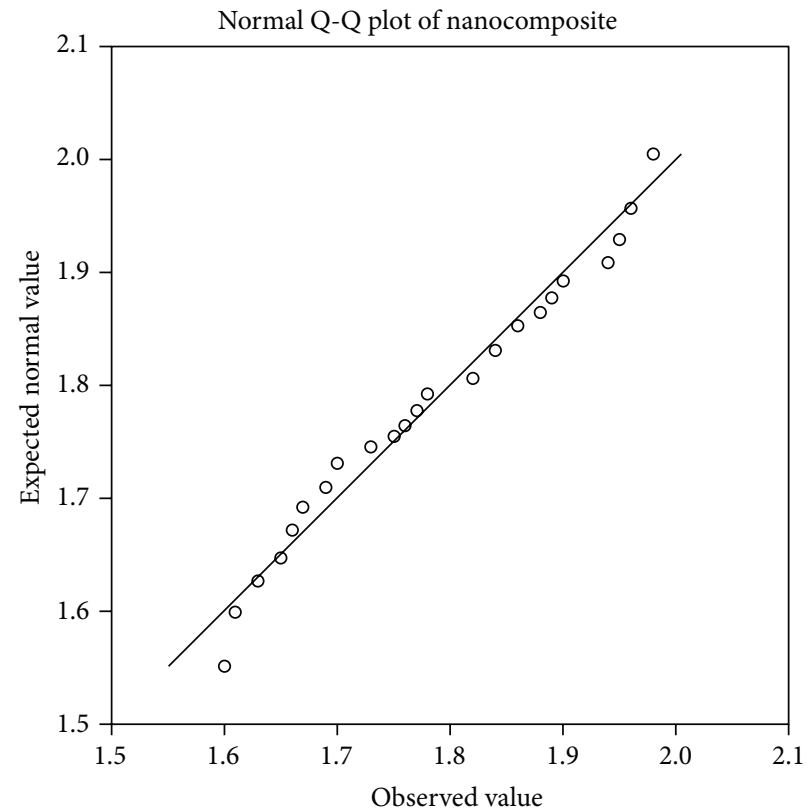

FIGURE 6: Normal probability curve of the fractal data distribution for the manganese-chromium bimetallic nanocomposite.

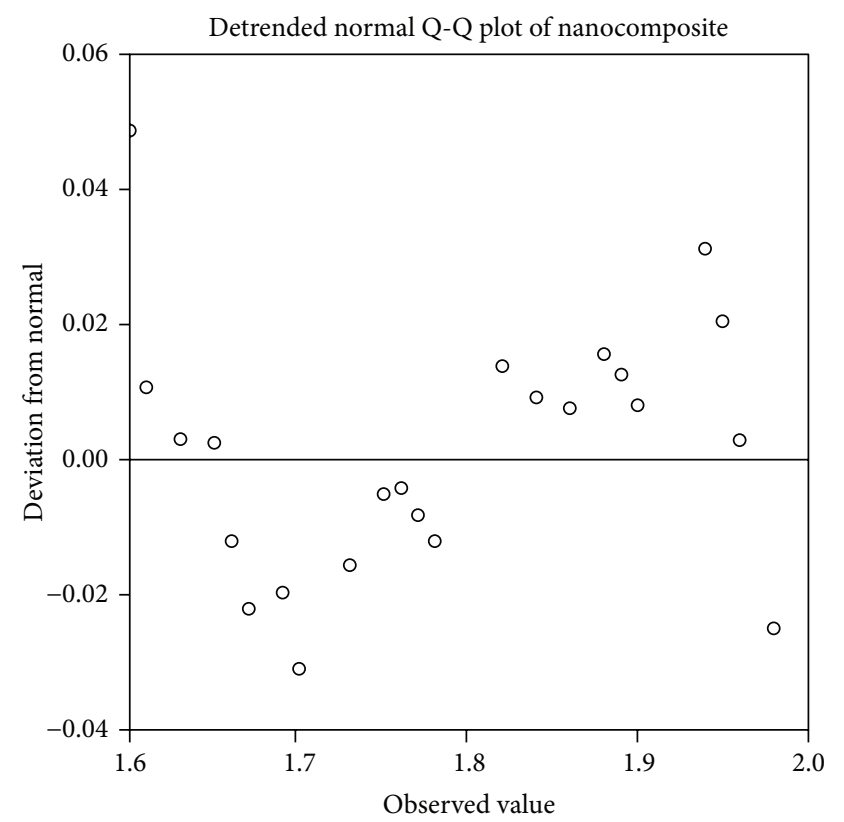

FIgURE 7: Residuals curve versus the values of fractal variables for the manganese-chromium bimetallic nanocomposite.

despite the uniform dispersion of the particles, their sizes and morphologies were different.

\section{Conflict of Interests}

The authors declare that there is no conflict of interests regarding the publication of this paper. 


\section{Acknowledgments}

The authors gratefully acknowledge the financial support from the Research Council of Imam Khomeini International University and Mollasadra student home research. The authors also warmly acknowledge the support of Shahrbano Maleki, Islamic Azad University, Ardabil Branch, Faculty of Science, chemistry group, for his M.S. degree thesis on inorganic chemistry. Daniel Glossman-Mitnik is a Researcher at CONACYT and CIMAV and acknowledges partial support from both institutions.

\section{References}

[1] M. Surender, B. Basu, and R. Balasubramaniam, "Wear characterization of electrodeposited Ni-WC composite coatings," Tribology International, vol. 37, no. 9, pp. 743-749, 2004.

[2] N. Sombatsompop, K. Sukeemith, T. Markpin, and N. Tareelap, "A new experimental apparatus of electro-codeposited system for Ni-WC composite coatings," Materials Science and Engineering A, vol. 381, no. 1-2, pp. 175-188, 2004.

[3] G. L. Povirk, A. Needleman, and S. R. Nutt, "An analysis of residual stress formation in whisker-reinforced Al-SiC composites," Materials Science and Engineering A, vol. 125, no. 2, pp. 129-140, 1990.

[4] G. L. Povirk, A. Needleman, and S. R. Nutt, "An analysis of the effect of residual stresses on deformation and damage mechanisms in AlSiC composites," Materials Science and Engineering A, vol. 132, pp. 31-38, 1991.

[5] Y.-L. Shen, A. Needleman, and S. Suresh, "Coefficients of thermal expansion of metal-matrix composites for electronic packaging," Metallurgical and Materials Transactions A, vol. 25, no. 4, pp. 839-850, 1994.

[6] L. L. Shaw and D. B. Miracle, "Effects of an interfacial region on the transverse behavior of metal-matrix composites-a finite element analysis," Acta Materialia, vol. 44, no. 5, pp. 2043-2055, 1996.

[7] P. J. Ward, H. V. Atkinson, P. R. G. Anderson et al., "Semi-solid processing of novel MMCs based on hypereutectic aluminiumsilicon alloys," Acta Materialia, vol. 44, no. 5, pp. 1717-1727, 1996.

[8] J. C. Michel and P. Suquet, "An analytical and numerical study of the overall behaviour of metal-matrix composites," Modelling and Simulation in Materials Science and Engineering, vol. 2, pp. 637-658, 1994.

[9] N. Ramakrishnan, "An analytical study on strengthening of particulate reinforced metal matrix composites," Acta Materialia, vol. 44, no. 1, pp. 69-77, 1996.

[10] D. B. Zahl, S. Schmauder, and R. M. McMeeking, "Mechanical behavior of residually stressed composites with ductile and brittle constituents," Modelling and Simulation in Materials Science and Engineering, vol. 2, pp. 267-276, 1994.

[11] M. Suéry, C. Teodosiu, and L. F. Menezes, “Thermal residual stresses in particle-reinforced viscoplastic metal matrix composites," Materials Science and Engineering A, vol. 167, no. 1-2, pp. 97-105, 1993.

[12] L. Khezami and R. Capart, "Removal of chromium(VI) from aqueous solution by activated carbons: kinetic and equilibrium studies," Journal of Hazardous Materials, vol. 123, no. 1-3, pp. 223-231, 2005.

[13] X. Guo, G. T. Fei, H. Su, and L. De Zhang, "High-performance and reproducible polyaniline nanowire/tubes for removal of
Cr(VI) in aqueous solution," The Journal of Physical Chemistry C, vol. 115, no. 5, pp. 1608-1613, 2011.

[14] S. R. Yeduru, A. Backen, S. Fähler, L. Schultz, and M. Kohl, "Transformation behaviour of freestanding epitaxial Ni-Mn-Ga films," Journal of Alloys and Compounds, vol. 577, no. 1, pp. S353S357, 2013.

[15] K. Hayashi, K. Miyata, F. Katsuki, T. Ishimoto, and T. Nakano, "Individual mechanical properties of ferrite and martensite in Fe-0.16 mass\% C-1.0 mass\% Si-1.5 mass\% Mn steel," Journal of Alloys and Compounds, vol. 577, no. 1, pp. S593-S596, 2013.

[16] Q. Li, M. Wang, and W. Gu, "Computer vision based system for apple surface defect detection," Computers and Electronics in Agriculture, vol. 36, no. 2-3, pp. 215-223, 2002.

[17] A. Hosseini, S. M. Jafari, H. Mirzaei, A. Asghari, and S. Akhavan, "Application of image processing to assess emulsion stability and emulsification properties of Arabic gum," Carbohydrate Polymers, vol. 126, pp. 1-8, 2015.

[18] D. H. De Boer and M. Stone, "Fractal dimensions of suspended solids in streams: comparison of sampling and analysis techniques," Hydrological Processes, vol. 13, no. 2, pp. 239-254, 1999.

[19] D. H. de Boer, "An evaluation of fractal dimensions to quantify changes in the morphology of fluvial suspended sediment particles during baseflow conditions," Hydrological Processes, vol. 11, no. 4, pp. 415-426, 1997.

[20] B. H. Kaye, "Image analysis techniques for characterizing fractal structures," in The Fractal Approach to Heterogeneous Chemistry: Surfaces, Colloids, Polymers, D. Avnir, Ed., Wiley, Chichester, UK, 1989.

[21] P. A. Burrough, "Fractal dimensions of landscapes and other environmental data," Nature, vol. 294, no. 5838, pp. 240-242, 1981.

[22] B. T. Milne, "Measuring the fractal geometry of landscapes," Applied Mathematics and Computation, vol. 27, no. 1, pp. 67-79, 1988.

[23] B. J. West and A. L. Goldberger, "Physiology in fractal dimensions," The Journal of American Science, vol. 75, pp. 354-365, 1987.

[24] C. J. Du and D. W. Sun, "Recent developments in the applications of image processing techniques for food quality evaluation," Trends in Food Science \& Technology, vol. 15, no. 5, pp. 230-249, 2004.

[25] R. J. Arsenault and M. Taya, "Thermal residual stress in metal matrix composite," Acta Metallurgica, vol. 35, no. 3, pp. 651-659, 1987.

[26] P. J. Withers, W. M. Stobbs, and O. B. Pedersen, "The application of the eshelby method of internal stress determination to short fibre metal matrix composites," Acta Metallurgica, vol. 37, no. 11, pp. 3061-3084, 1989.

[27] B. B. Mandelbrot, The Fractal Geometry of Nature, W. H. Freeman and Company, New York, NY, USA, 1982.

[28] J.-F. Gouyet and B. B. Mandelbrot, Physics and Fractal Structures, Springer, New York, NY, USA, 1996.

[29] K. Falconer, Fractal Geometry: Mathematical Foundations and Applications, John Wiley \& Sons, New York, NY, USA, 2003.

[30] X. C. Jin, S. H. Ong, and Jayasooriah, "A practical method for estimating fractal dimension," Pattern Recognition Letters, vol. 16, no. 5, pp. 457-464, 1995.

[31] A. K. Bisoi and J. Mishra, "On calculation of fractal dimension of images," Pattern Recognition Letters, vol. 22, no. 6-7, pp. 631637, 2001. 
[32] J. Zhang, R. Zhang, B. Hu, and S. Bai, "Fractal image processing and analysis by programming in MATLAB," in Proceedings of the 8th WSEAS International Conference on Mathematics and Computers in Biology and Chemistry, Vancouver, Canada, June 2007.

[33] B. Mandelbrot, "How long is the coast of Britain? Statistical selfsimilarity and fractional dimension," Science Magazine, vol. 156, no. 3775, pp. 636-638, 1967.

[34] E. Perfect, "Estimating soil mass fractal dimensions from water retention curves," Geoderma, vol. 88, no. 3-4, pp. 221-231, 1999.

[35] M. Borkovec, W. de Paris, and R. Peikert, "The fractal dimension of the Apollonian sphere packing," Fractals, vol. 2, no. 4, pp. 521526, 1994

[36] Z. Khanbabaei, A. Karam, and G. Rostamizad, "Studying relationships between the fractal dimension of the drainage basins and some of their geomorphological characteristics," International Journal of Geosciences, vol. 4, pp. 636-642, 2013.

[37] P. Parsons-Wingerter, B. Lwai, M. C. Yang et al., "A novel assay of angiogenesis in the quail chorioallantoic membrane: stimulation by bFGF and inhibition by angiostatin according to fractal dimension and grid intersection," Microvascular Research, vol. 55, no. 3, pp. 201-214, 1998.

[38] E. Guérin and E. Tosan, "Fractal inverse problem: approximation formulation and differential methods," Fractals in Engineering: New Trends in Theory and Applications, pp. 271$285,2005$.

[39] M. K. Biswas, T. Ghose, S. Guha, and P. K. Biswas, "Fractal dimension estimation for texture images: a parallel approach," Pattern Recognition Letters, vol. 19, no. 3-4, pp. 309-313, 1998.

[40] The MathWorks, MATLAB-Statistics Toolbox, Release 2012, The MathWorks, Natick, Mass, USA, 2012.

[41] SPSS, SPSS Statistics for Windows, Version 17.0, SPSS, 2008.

[42] G. F. Voort, ASM Handbook, Volume 9: Metallography and Microstructures, ASM International, Materials Park, Ohio, USA, 2004.

[43] L. Wojnar, Image Analysis, Applications in Materials Engineering, CRC Press, Boca Raton, Fla, USA, 1999. 

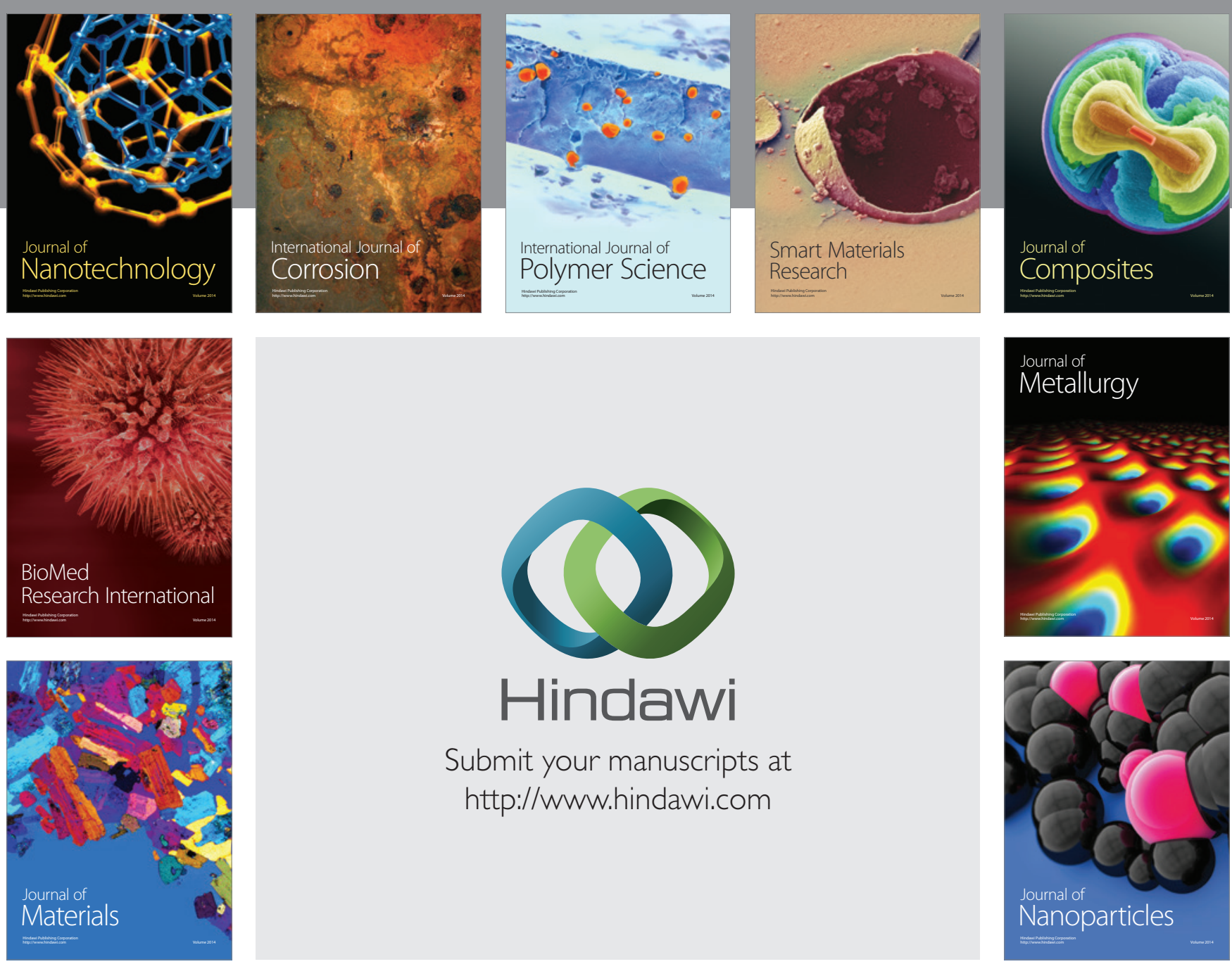

Submit your manuscripts at http://www.hindawi.com
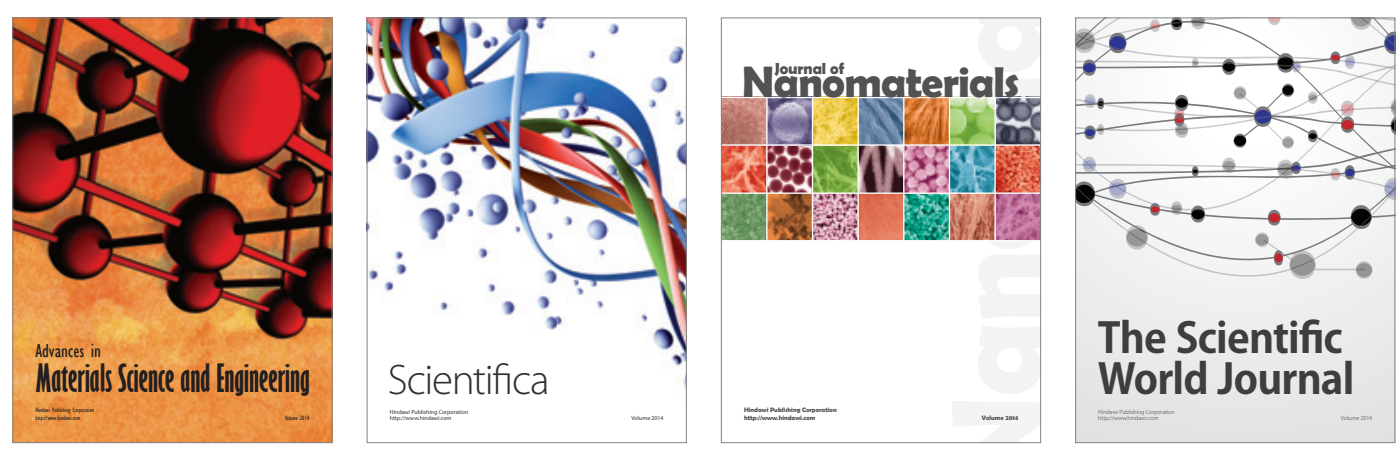

\section{The Scientific World Journal}
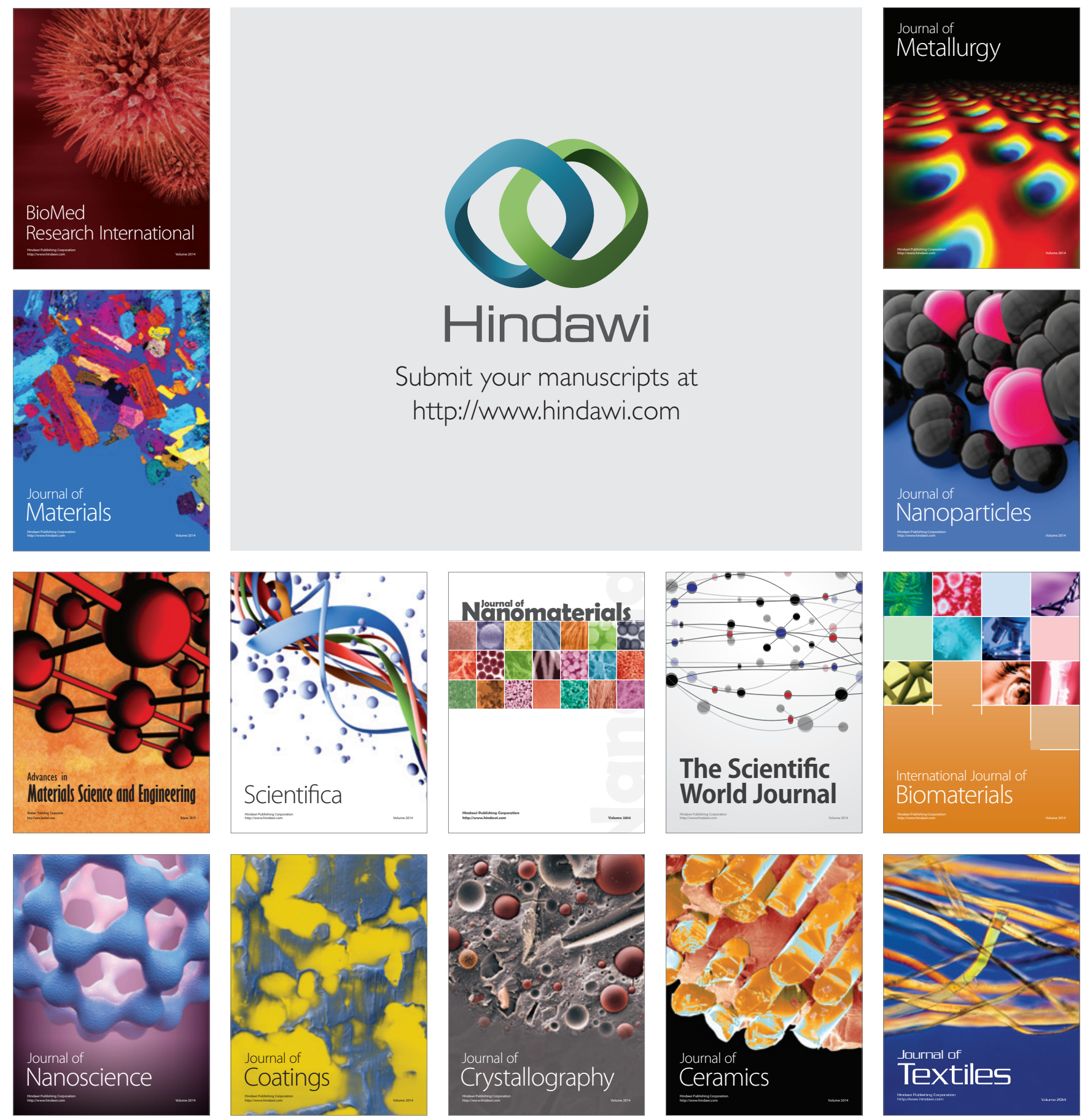